\title{
KINETICS OF REACTIVE WETTING
}

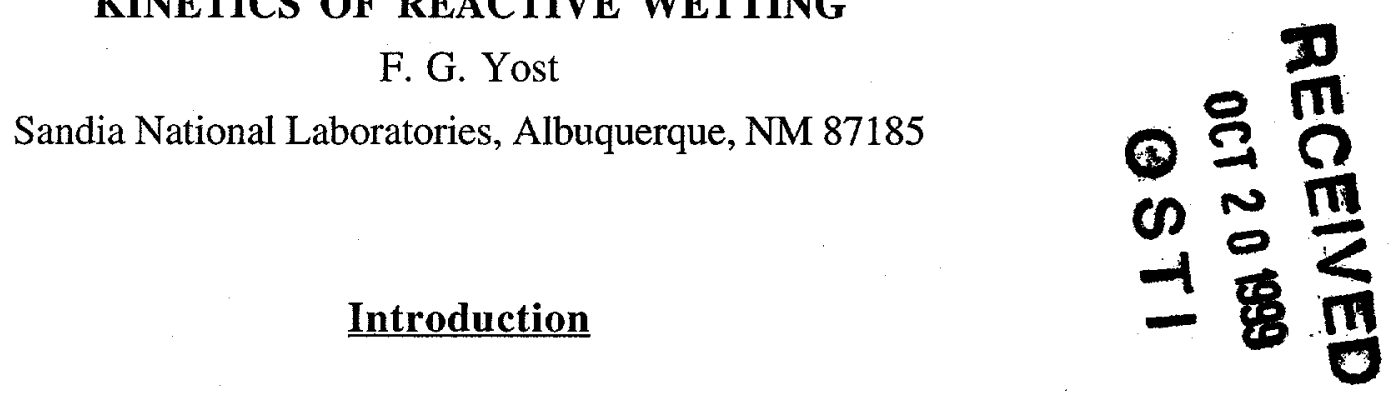

The importance of interfacial processes in materials joining has a long history. A significant amount of work has suggested that processes collateral to wetting can affect the extent of wetting and moderate or retard wetting rate. Even very small additions of a constituent, known to react with the substrate, cause pronounced improvement in wetting and are exploited in braze alloys, especially those used for joining to ceramics [1,2]. The wide diversity of processes, such as diffusion, chemical reaction, and fluxing, and their possible combinations suggest that various rate laws should be expected for wetting kinetics depending on the controlling processes. These rate laws are expected to differ crucially from the standard fluid controlled wetting models found in the literature [3-5]. Voitovitch et al. [6] and Mortensen et al. [7] have shown data that suggests diffusion control for some systems and reaction control for others. They also presented a model of wetting kinetics controlled by the diffusion of a constituent contained by the wetting fluid.

In the following a model will be constructed for the wetting kinetics of a small droplet of metal containing a constituent that diffuses to the wetting line and chemically reacts with a flat, smooth substrate. The model is similar to that of Voitovitch et al. and Mortensen et al. but incorporates chemical reaction kinetics such that the result contains both diffusion and reaction kinetics. The model is constructed in the circular cylinder coordinate system, satisfies the diffusion equation under conditions of slow flow, and considers diffusion and reaction at the wetting line to be processes in series. This is done by solving the diffusion equation with proper initial and boundary conditions, computing the diffusive flux at the wetting line and equating this to both the convective flux and reaction flux. This procedure is similar to equating the current flowing in components of a series circuit. The wetting rate will be computed versus time for a variety of diffusion and reaction conditions. A transition is observed from nonlinear (diffusive) to linear (reactive) behavior as the control parameters (such as the diffusion coefficient) are modified. This is in agreement with experimental observations [5,6]. The adequacy of the slow flow condition, used in this type of analysis, is discussed and an amended procedure is suggested. 


\section{DISCLAIMER}

This report was prepared as an account of work sponsored by an agency of the United States Government. Neither the United States Government nor any agency thereof, nor any of their employees, make any warranty, express or implied, or assumes any legal liability or responsibility for the accuracy, completeness, or usefulness of any information, apparatus, product, or process disclosed, or represents that its use would not infringe privately owned rights. Reference herein to any specific commercial product, process, or service by trade name, trademark, manufacturer, or otherwise does not necessarily constitute or imply its endorsement, recommendation, or favoring by the United States Government or any agency thereof. The views and opinions of authors expressed herein do not necessarily state or reflect those of the United States Government or any agency thereof. 


\section{DISCLAIMER}

Portions of this document may be illegible in electronic image products. Images are produced from the best available original document. 


\section{The Model}

When two or more distinct processes operate simultaneously in chemical and metallurgical reactions they often appear in series (coupled) or in parallel (competitive) combinations. An example of a series process is given by oxidation of silicon. Oxidant species must first diffuse through a thin oxide layer and then must react with silicon at the oxide/silicon interface [8]. Diffusion through a polycrystalline material provides an example of a parallel process. Grain boundary diffusion and volume diffusion can occur at comparable rates and jointly provide the total flux at any given plane perpendicular to the diffusion direction [9]. Wetting under conditions involving fluid flow, diffusion, and chemical reaction is viewed as a series combination of processes in which the slowest of these would be rate controlling. If the liquid is capable of wetting the bare substrate (without reaction), wetting kinetics are often determined by liquid surface tension and viscosity. The rate of wetting by metallic droplets under conditions of fluid flow control has been shown to be extremely rapid [10] compared to that in situations involving diffusion and reaction control. If these three processes act in series, fluid flow kinetics can safely be ignored. Under these circumstances the liquid does not wet the substrate until the diffusing species, A, reacts with a substrate constituent, B, to produce a product, $\mathrm{C}$. In this situation the wetting liquid advances from one metastable capillary state to another and when spreading ceases may still be in metastable capillary equilibrium.

The model is constructed in the circular cylinder coordinate system, does not treat the droplet shape, and views the diffusion and reaction processes as one dimensional (radial). The initial condition $(\mathrm{t}=0)$ is that the concentration of the diffusing/reacting species is $C(r, 0)=C_{o}$ while the boundary condition is $C_{a}$ at $r=r(t)$ where $r$ is radial dimension and $r(t)$ is the location of the wetting line. The wetting rate, $\dot{r}(t)$, is the time derivative of $r(t)$ and the condition for quasi-equilibrium or slow flow is

$$
\dot{r}(t) \ll \frac{D}{r(t)}
$$

where $D$ is the interdiffusion coefficient for the liquid droplet. For reaction to proceed, the concentration at the wetting line, $C_{a}$, must be slightly greater than, $C_{e}$, which would be that concentration in chemical equilibrium with the substrate. A solution to the diffusion equation satisfying these conditions is [11]

$$
C(r, t)=C_{a}+\frac{2\left(C_{o}-C_{a}\right)}{r(t)} \sum_{n=1}^{\infty} e^{-D \lambda_{n}^{2} t} \frac{J_{0}\left[r \lambda_{n}\right]}{\lambda_{n} J_{1}\left[r(t) \lambda_{n}\right]}
$$

where $r(t) \lambda_{n}$ define roots of the Bessel function $J_{0}$ [11].

A flux balance at the wetting line yields 


$$
C_{a} \dot{r}(t)=\int_{0}^{\theta(t)} j_{d}(\phi, r) d \phi=\mu\left(C_{a}-C_{e}\right)
$$

where $j_{d}(\phi, r)$ is diffusive flux, as shown in Fig. 1, and $\mu$ is sometimes referred to as the interface transfer coefficient [12] and has units of velocity. The diffusive flux can be written

$$
j_{d}(\phi, r)=-\left.\cos (\phi) D \frac{\partial C}{\partial r}\right|_{r=r(t)}
$$

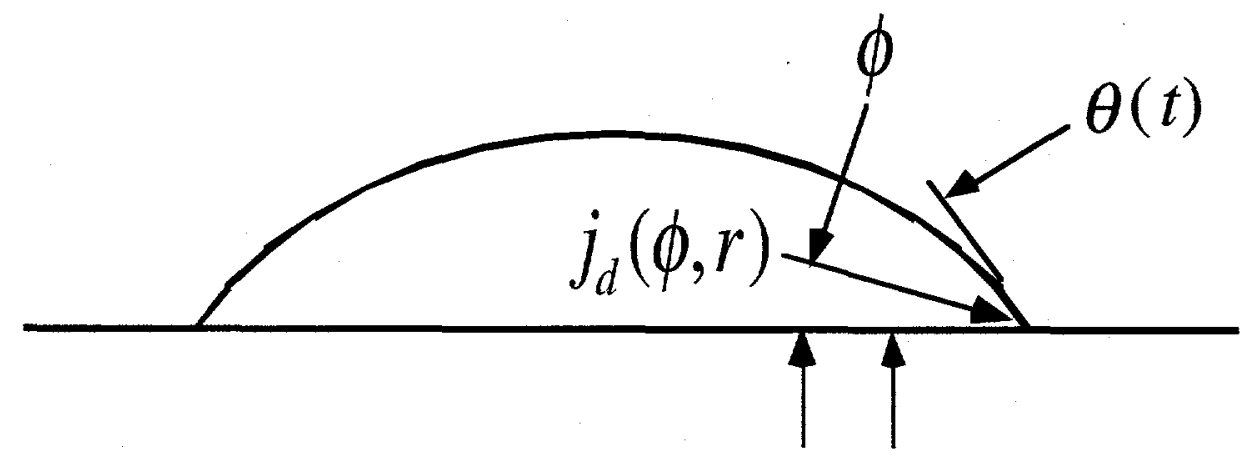

Figure 1. Illustration of the diffusive flux as a function of angle, $\phi$. The total flux is the integral of over $\phi$ from 0 to $\theta(t)$.

Performing the operations indicated in eqs. (2) and (3) yields

$$
C_{a} \dot{r}(t)=\mu\left(C_{a}-C_{e}\right)
$$

and

$$
\mu\left(C_{a}-C_{e}\right)=\frac{2\left(C_{o}-C_{a}\right) D}{r(t)} F(t) \sin \theta(t)
$$

where

$$
F(t)=\sum_{n=1}^{\infty} e^{-D \lambda_{n}^{2} t}
$$

Solving eq. (5) for $C_{a}$ and substituting into eq. (4) yields the equation of motion for the wetting line

$$
\dot{r}(t)=\frac{\frac{2 D}{r(t)}\left(\frac{C_{o}}{C_{e}}-1\right) F(t) \sin \theta(t)}{1+\frac{2 D}{r(t) \mu} F(t) \sin \theta(t) \frac{C_{o}}{C_{e}}} .
$$

The temporal contact angle, $\theta(t)$, in equation 6 is obtained by knowing the initial droplet volume given as

$$
V_{o}=\frac{\pi r^{3}(t)}{6} \tan \frac{\theta(t)}{2}\left(3+\tan ^{2} \frac{\theta(t)}{2}\right)
$$

and solving for $\theta(t)$. Note that the liquid volume is considered constant which is tantamount to assuming that the reaction consumes very little liquid during the spreading process. It can be seen 
from eq. (6) that under conditions of reaction control $\left(\frac{D}{\mu}>>1\right)$ the wetting rate is constant and given by

$$
\dot{r}(t)=\mu\left(1-\frac{C_{e}}{C_{o}}\right)
$$

while under diffusion control $\left(\frac{D}{\mu} \ll 1\right)$ the wetting rate is

$$
\dot{r}(t)=\frac{2 D}{r(t)}\left(\frac{C_{o}}{C_{e}}-1\right) F(t) \sin \theta(t) .
$$

It can be seen from eq. 8a that reaction control will yield linear spreading kinetics. Under diffusion control the kinetics, given by eq. $8 \mathrm{~b}$, are not parabolic due to the factors $F(t)$ and $\sin \theta(t)$.

With an assumed initial volume $V_{o}=0.1 \mathrm{~cm}^{3}$ equations 6 and 7 were solved for the initial conditions $\theta(0)=90^{\circ}$ and $r(0)=\left(\frac{3 V_{o}}{2 \pi}\right)^{1 / 3}$. The diffusion coefficient was taken as $D=4.0^{*} 10^{-5} \mathrm{~cm}^{2} / \mathrm{s}[6]$ and the equilibrium atom fraction of reactant species was assumed to be $2 * 10^{-2}$ for all calculations. The equations were solved for $r(t)$ and $\theta(t)$ with three values of $\mu$ and three values of $\frac{C_{o}}{C_{e}}=\frac{X_{o}}{X_{e}}$ (assuming concentration independent molar volume) where $X_{o}$ and $X_{e}$ are initial and equilibrium atom fractions of reactant. Figures $2 \mathrm{a}$ and $2 \mathrm{~b}$ show that under reaction control $(D / \mu>>1)$ the kinetics of spreading increase with increasing $\mu$ which could have been anticipated from eq. 8a. With the various chosen parameters the reaction kinetics appear quite linear even at $D / \mu=1$. Similarly, the contact angle drops more rapidly as $\mu$ is increased, but for $D / \mu=1$ the kinetics are decidedly nonlinear. For larger values of $\mu$, the plot is linear over the time span of the calculation. 


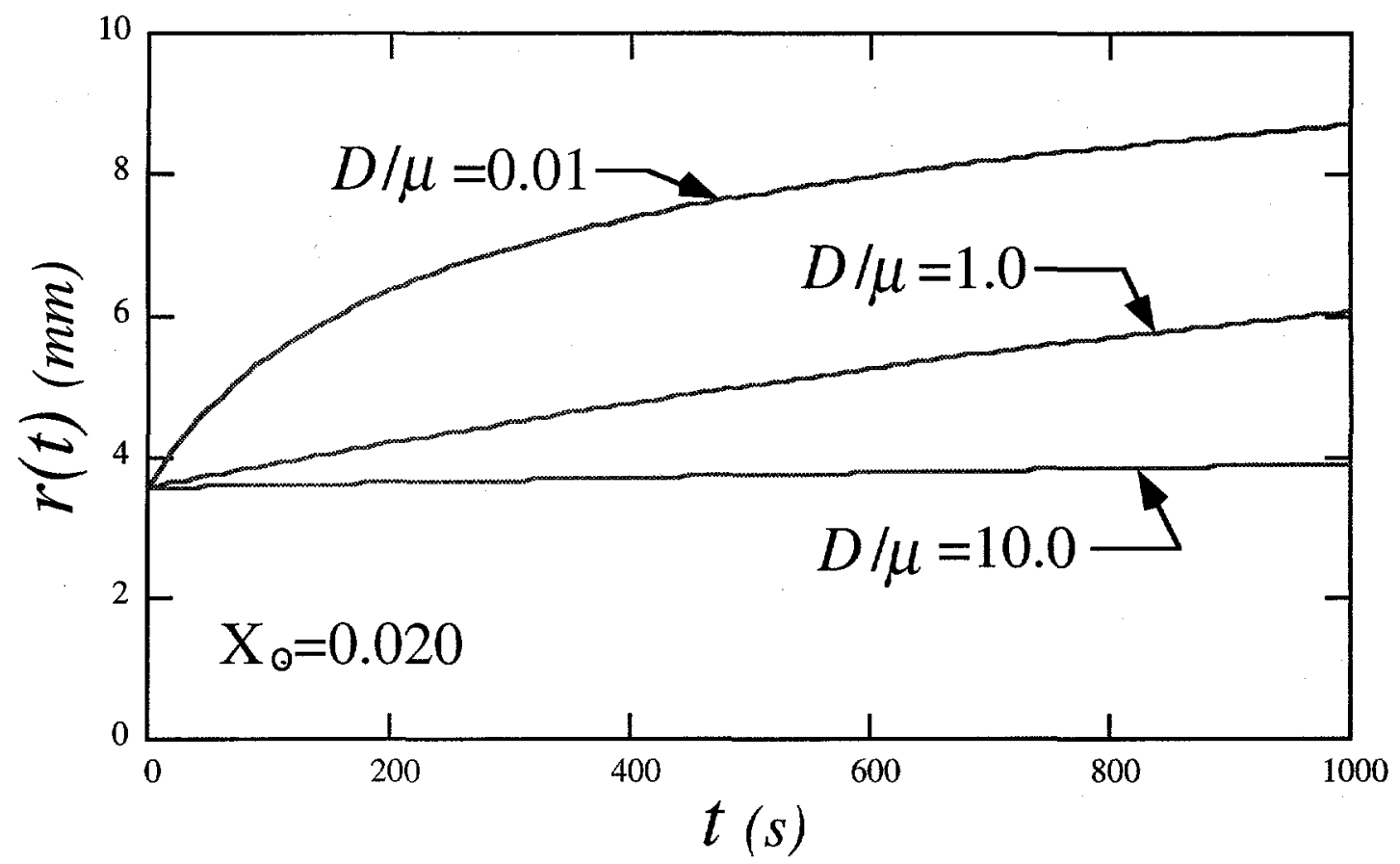

Figure 2a. The radius of a spreading droplet versus time under reaction control and mixed diffusion and reaction control.

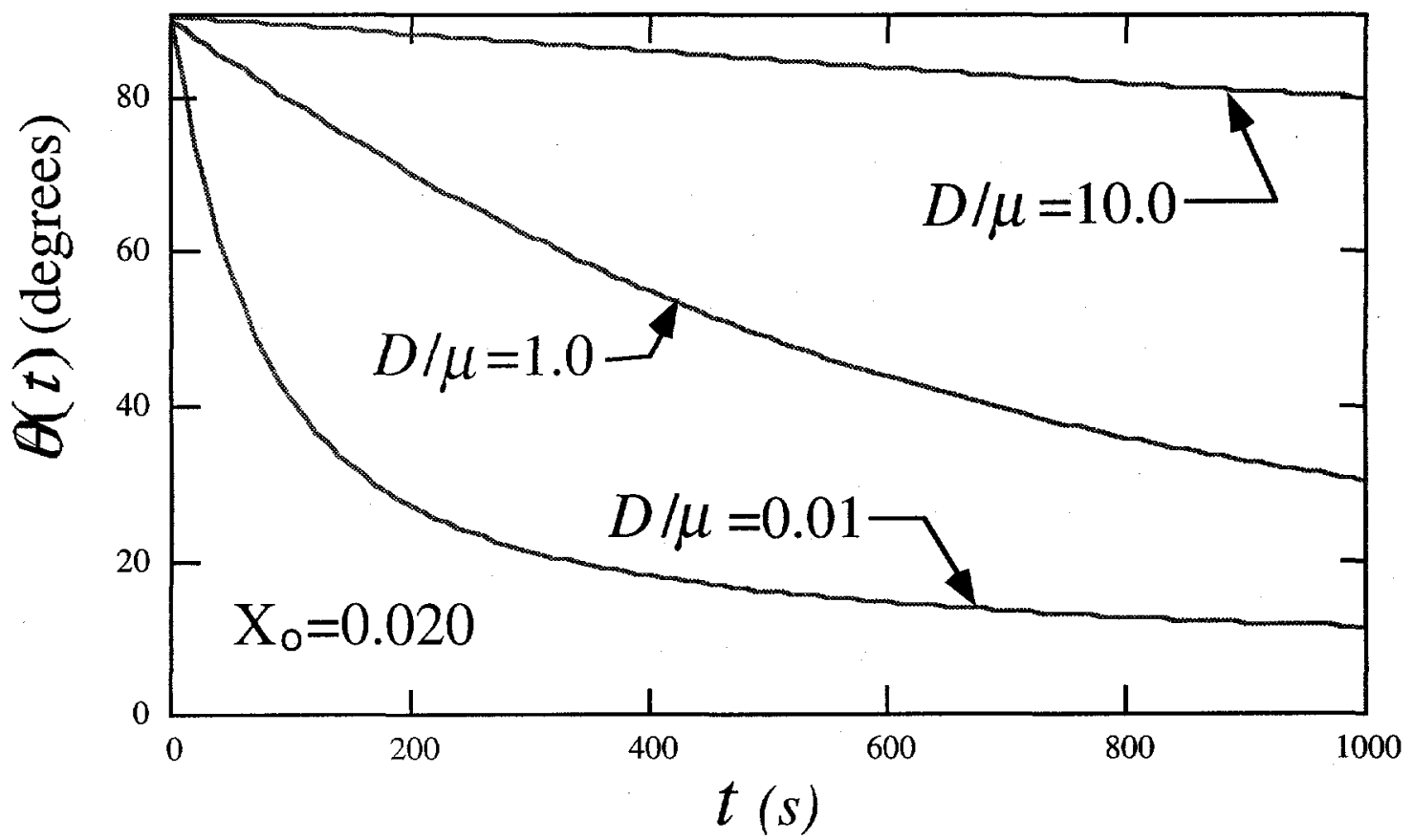

Figure $2 \mathrm{~b}$. The temporal contact angle of a spreading droplet versus time under reaction control and mixed diffusion and reaction control. 
When diffusion of reactant is controlling, the rate and extent of spreading increases as the initial concentration of reactant is increased as shown in Fig. 3a. This is in agreement with experimental behavior [6]. When plotted on logarithmic axes the plots are quite linear but have slopes that differ from parabolic behavior and are a function of initial concentration of reactant. Kinetics obtained from solutions of eqs. 6 and 7 are independent of $\mu$, when $D / \mu<<1$, which could have been predicted from eq. $8 \mathrm{~b}$. Figure $3 \mathrm{~b}$ shows the temporal contact angle decreasing rapidly towards an asymptote. On logarithmic axes these plots are also quite linear with slopes depending on the initial concentration of reactant. The model is capable of predicting the final contact angle but this represents no useful material property as it is a metastable configuration.

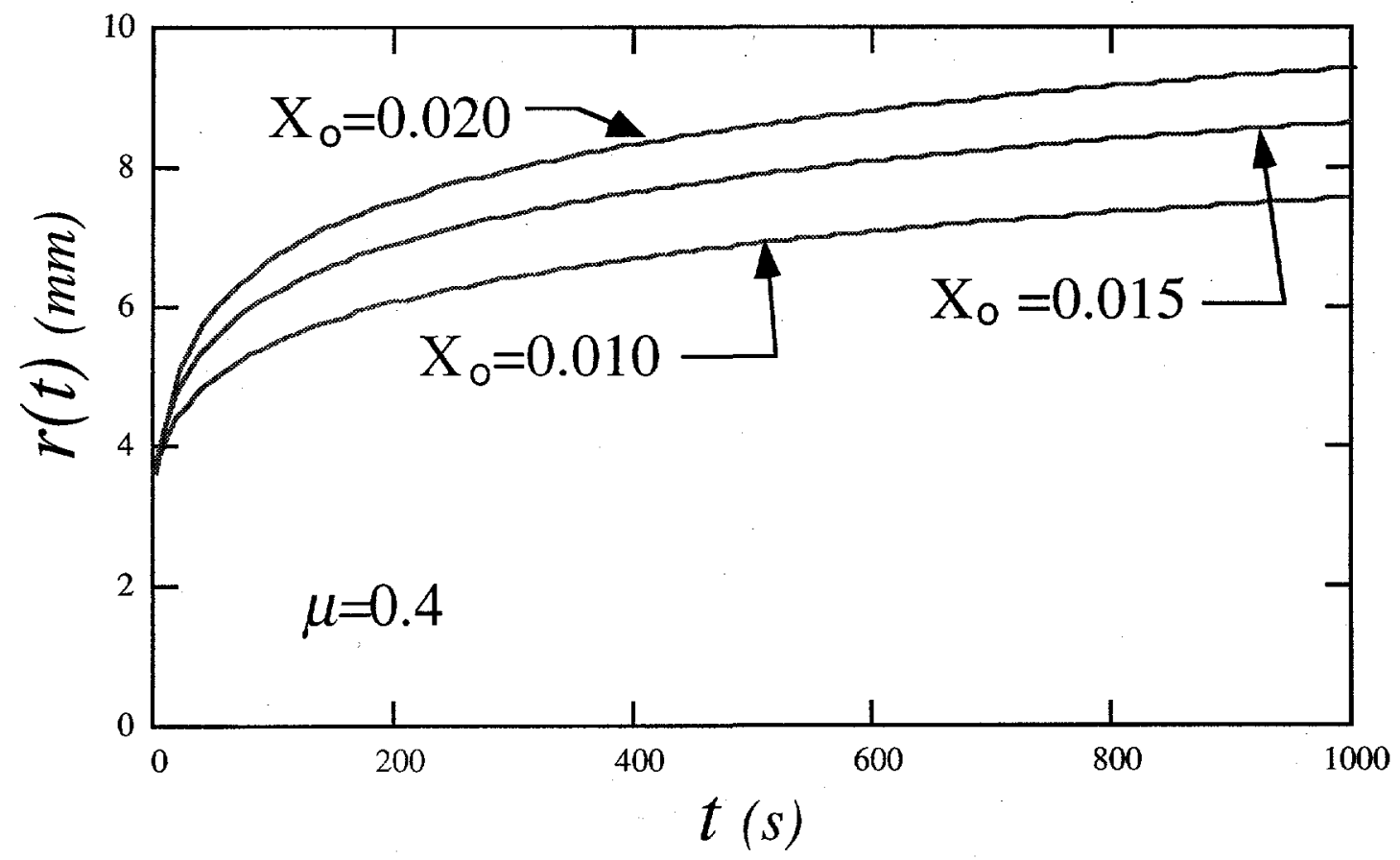

Figure 3a. The radius of a spreading droplet versus time under diffusion controlled conditions. The kinetics cannot be characterized as parabolic. 


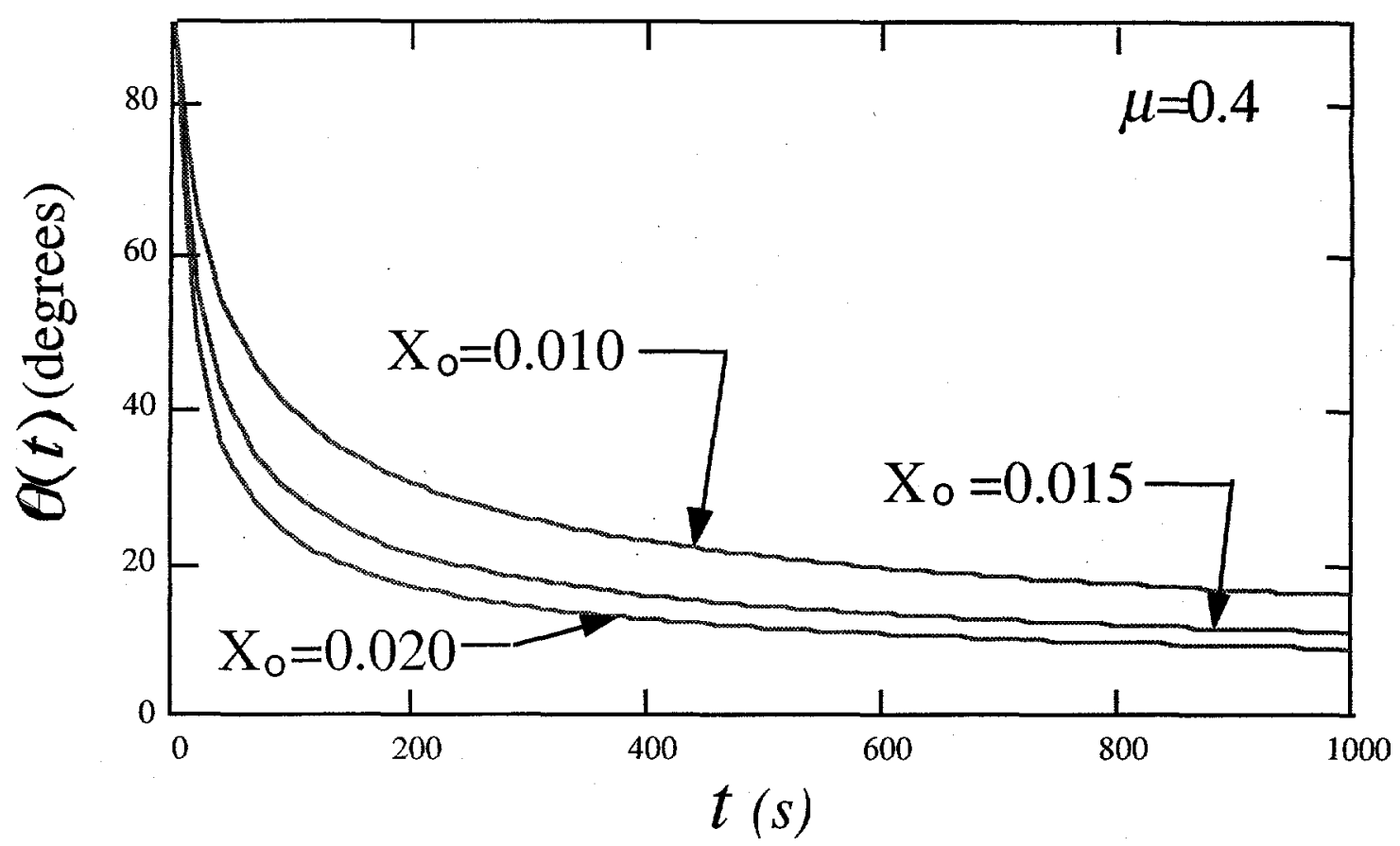

Figure $3 \mathrm{~b}$. The temporal contact angle of a spreading droplet versus time under diffusion control. The asymptotic limit suggested by these plots is not a capillary equilibrium configuration.

\section{Discussion and Conclusions}

A model for the kinetics of spreading under conditions of mixed reaction and diffusion control has been constructed. The model is similar to those of Voitovitch et al. [6] and Mortensen et al. [7] and employs the concept of processes in series used by Deal and Grove [8] for oxidation of Si. Under diffusion controlled conditions the wetting kinetics are not parabolic due to the factors $F(t)$ and $\sin \theta(t)$ in eq. $8 \mathrm{~b}$. The model exhibits a transition from nonlinear to linear behavior in time as $D / \mu$ approaches 1. In addition, it shows more rapid and more extensive wetting as the initial concentration of reactant is increased. To obtain an analytical solution in the circular cylinder coordinate system it was assumed that wetting rate is slow and obeys the inequality

$$
\dot{r}(t) \ll \frac{D}{r(t)} .
$$

The diffusion coefficient cited by Voitovitch et al. [6], for transition metals in copper at $1373 \mathrm{~K}$, was $D=4^{*} 10^{-3} \mathrm{~mm}^{2} / \mathrm{s}$. This data is supported by that measured by Lozovskii et al. [13] for $\mathrm{Si}$ in $\mathrm{Cu}$ at the same temperature. Figure 2 of Voitovitch [6] shows wetting kinetics of $\mathrm{Cu}-\mathrm{Cr}$ alloys on vitreous carbon substrates that are suggestive of diffusion control. With these kinetics and the above diffusion coefficient it can be shown that the above inequality is not obeyed. On the contrary, their Fig. 6, for wetting of $\mathrm{Cu}-\mathrm{Si}$ alloys on vitreous carbon, is suggestive of reaction control and does satisfy the 
inequality. A similar analysis of Figs. 1 and 2 from Drevet et al. [14] yields the same conclusion. Their Figure 1, for wetting of $\mathrm{Cu}$-Ti alloys on alumina, is suggestive of diffusion control but, with the diffusion coefficient as was used above, the inequality is not obeyed. Their Figure 2, for wetting of Al on pyrocarbon is suggestive of reaction control, and with the diffusion coefficient $\left(9.7 * 10^{-5} \mathrm{~cm}^{2} / \mathrm{s}\right)$ measured by Levin [15], satisfies the inequality. These results imply that the slow flow or quasiequilibrium assumption, made to simplify analysis such that analytical solutions to the diffusion equation could be found, are inadequate except for reaction controlled flow conditions. To compose a realistic model for diffusion controlled wetting, transformation to a coordinate system moving with the wetting line seems necessary. This involves numerical solutions to the resulting diffusion equation and will be addressed in a separate report. Finally, it should be emphasized that the reaction mechanism assumed in the model presented here is just one of many that can ultimately control wetting kinetics. For example, a gaseous environment may react with the substrate and be the controlling process. It would be difficult to incorporate all reaction possibilities in one model.

\section{Acknowledgment}

This work was performed at Sandia National Laboratories, which is supported by the United States Department of Energy under contract number DE-AC04-94AL85000. Sandia is a multi-program laboratory operated by Sandia Corporation, a Lockheed Martin Company, for the United States Department of Energy. 


\section{References}

1. M. Humenik, Jr. and W. D. Kingery, J. Amer. Ceramic Soc., 37, 18 (1954).

2. P. Kritsalis, L. Coudurier, and N. Eustathopoulos, J. Mater. Sci., 26, 3400 (1991).

3. T. D. Blake and J. M. Haynes, J. Colloid Interface Sci., 30, 421 (1969).

4. P. G. de Gennes, Rev. Mod. Phys. 57, 827 (1985).

5. P. Ehrhard and S. H. Davis, J. Fluid Mech., 229, 365 (1991).

6. R. Voitovitch, A. Mortensen, F. Hodaj, and N. Eustathopoulos, Acta Mat., 47, 1117 (1999).

7. A. Mortensen, B. Drevet, and N. Eustathopoulos, Scripta Mat., 36, 645 (1997).

8. B. E. Deal and A. S. Grove, J. Appl. Phys., 36, 3770 (1965).

9. P. G. Shewmon, Diffusion in Solids, McGraw-Hill Book Company, New York, NY (1963).

10. F. G. Yost, F. M. Hosking, and D. R. Frear, in The Mechanics of Solder Alloy Wetting and Spreading, edited by F. G. Yost, F. M. Hosking, and D. R. Frear, Van Nostrand Reinhold, New York, NY (1993).

11. H. S. Carslaw and J. C. Jaeger, Conduction of Heat in Solids, 2nd Ed., Clarendon Press, Oxford, UK, p.199 (1959).

12. J. S. Kirkaldy and D. J. Young, Diffusion in the Solid State, The Institute of Metals, London, UK, p.52 (1987).

13. V. N. Losovskii, B. M. Gurov, and V. S. Zurnadzhyan, Russian Journal of Physical Chemistry, 60, (11), 1719 (1986).

14. B. Drevet, K. Landry, P. Vikner, and N. Eustathopoulos, Scripta Materialia, 35, 1265 (1996).

15. E. S. Levin, Russian Metallurgy, 5, 54, (1971). 